\title{
Frequency modeling of wind power fluctuation and the application on power systems
}

\author{
Lin, Jin; Sun, Yuan-zhang; Sørensen, Poul Ejnar; Li, Guo-jie; Li, Xiong
}

Published in:

Conference proceedings

Publication date:

2010

Document Version

Publisher's PDF, also known as Version of record

Link back to DTU Orbit

Citation (APA):

Lin, J., Sun, Y., Sørensen, P. E., Li, G., \& Li, X. (2010). Frequency modeling of wind power fluctuation and the application on power systems. In Conference proceedings

\section{General rights}

Copyright and moral rights for the publications made accessible in the public portal are retained by the authors and/or other copyright owners and it is a condition of accessing publications that users recognise and abide by the legal requirements associated with these rights.

- Users may download and print one copy of any publication from the public portal for the purpose of private study or research.

- You may not further distribute the material or use it for any profit-making activity or commercial gain

- You may freely distribute the URL identifying the publication in the public portal

If you believe that this document breaches copyright please contact us providing details, and we will remove access to the work immediately and investigate your claim. 


\title{
Frequency modeling of wind power fluctuation and the application on power systems
}

\author{
LIN Jin, SUN Yuan-zhang, Senior Member, IEEE, Poul Sørensen, Senior Member, IEEE, LI Guo-jie, \\ and LI Xiong
}

\begin{abstract}
System imbalance due to power fluctuation is the first concern of power grid with large scale wind power integration. Traditional studies are limited in time domain, where the modeling is not convenient enough. In this paper, a model based on frequency domain is proposed. This model is more precise, and capable to take some important factors, such as the layout of wind farm, into account. This paper also presents a fast engineering implementation of this model, which can be applied on different power system studies. The case studies show this model can perfectly evaluate the ramp rate and reserve requirement of power systems, no matter under normal or extreme weather condition, therefore the validity and flexibility of this model get proved.
\end{abstract}

Index Terms-Power fluctuation, Frequency Domain, Reserve Requirement, Ramp Requirement

\section{INTRODUCTION}

$\mathrm{W}$ ITH the development of society, more wind power is integrating into power grid to meet the emissionreduction demand of low-carbon economy. However, the first concern of system operation is to maintain the balance between the generation and demand, which is against by the large power fluctuation from wind farm. Thus, it is necessary to develop a wind power fluctuation model for fundamental study and industry application, in order to support the security operations of power systems.

This subject has attracted a lot of worldwide efforts since the start of the wind energy expansion in power grid. Before 2002, it has been concluded from empirical observations that the power fluctuation of wind farms can be reduced if wind turbines disperse on a wider area[1].In contrast, a concentrated wind farm layout can bring more fluctuations of power production, especially in the minute-minute scale[2][3]. [3] uses a macro-model in terms of vector diagrams to explain this "Effect of geographical dispersion".

Wind power fluctuation model has also been applied on

This work was supported by the Chinese National Natural Science Funds (50977050, 50823001).

LIN Jin is a PhD student from State Key Laboratory of Power Systems, Department of Electrical Engineering, Tsinghua University, Beijing 100084, P. R. China. Now he is a visiting student in Risø, DTU. (e-mail: linjin03@mails.tisnghua.edu.cn).

SUN Yuan-zhang is the professor of Tsinghua University. He is also the dean of electrical engineering, Wuhan University. (yzsun@mail.tsinghua.edu.cn).

P. Sørensen is the professor of Wind Energy Division, Risø, DTU. (email: posq@risoe.dtu.dk). power system industry applications. [4][5] use ARMA fluctuation model to study the reliability impact of the wind farm integration on power systems. By assuming the wind power fluctuation fitting normal distribution, [6][7] study the system reserve requirement and the unit commitment strategy of the system with significantly high wind penetration. [8][9] study the frequency deviation caused by power fluctuation to estimate the highest wind power penetration.

Although these previous studies contribute to the progresses of wind power integration, the utilized wind power fluctuation models are still limited in time domain, where many important factors, such as the dynamics of wind turbine and the layout of wind farm, are not possible to be considered. That may misestimate the study results, since the requirements from simulation condition matrix in [10] cannot be completely satisfied.

In this paper, a model based on frequency domain is proposed. The stochastic wind speed is modeled by PSD (Power Spectrum Density), so the dynamics and coherences of wind turbines can be fully taken into account. Since the frequency region can be extended unlimitedly, it is possible to simulate the power fluctuation from seconds to hours. Therefore this model is flexible enough to be applied on different industry applications of power system.

The design of this model is supported by the long-term research works [11][12][13][14] of Risø, Denmark. It is originally used to evaluate the power quality of single wind turbine[11] and then extended to simulate the power fluctuation from one wind farm[12][13] or even one region[14]. Tsinghua University also joins the research, contributing on the improvements of frequency-time transitions and the applications on power system studies [15][16]. This paper organizes all these works together: In Section II, the mathematical background is introduced; Section III presents the basic structure of this model, then Section IV gives a fast engineering implementation; The applications on power systems are studied in Section V, which show this model can perfectly evaluate the ramp rate and reserve requirement of power systems, no matter under normal or extreme weather condition. Conclusions are finally presented in Section VI.

\section{MAthematical BACKGRound of FREQUENCY MODELing OF FLUCTUATION}

The core of frequency modeling of fluctuation is PSD (Power Spectrum Density), which is based on the theory of 
wide-sense stationarity (WSS). This type of stationary process has been widely used in engineering, for instance to model airflow turbulence or wave fluctuation. The feature of WSS is the successive random variables are correlated due to the "inertial" of the process, and the correlation is assumed to keep constant within a certain time region. Obviously, the wind power fluctuation just befits this process.

This kind of "inertial" can be modeled in time domain by a term of autocorrelation function, which satisfies the restriction of the mathematical definition of WSS as (1) and (2):

A continuous-time WSS $x(t)$ has the following restrictions on its mean function,

$$
E(x(t))=m_{x}(t)=m_{x}(t+\tau), \forall \tau \in R
$$

and autocorrelation function,

$$
\begin{aligned}
E\left(x\left(t_{1}\right) x\left(t_{2}\right)\right)= & R_{x}\left(t_{1}, t_{2}\right)=R_{x}\left(t_{1}+\tau, t_{2}+\tau\right) \\
& =R_{x}\left(t_{1}-t_{2}, 0\right), \forall \tau \in \mathrm{R}
\end{aligned}
$$

The first property (1) implies that the mean function $m_{x}(t)$ must be constant. The second property (2) implies that the correlation function depends only on the difference between $t_{1}$ and $t_{2}$ and only needs to be indexed by one variable rather than two variables. Thus, the abbreviation $R_{x}(\tau)$ where $\tau=t_{1}-t_{2}$ is commonly chosen as the instead of writing $R_{x}\left(t_{1}-t_{2}, 0\right) . R_{x}(\tau)$ depicts the "inertial" of process, so then it plays an important role to describe the statistics of WSS.

In most of engineering cases, frequency domain is usually more convenient and powerful than time domain. For this paper's study, PSD $S(f)$ is introduced in to denote the statistical features of stochastic process in frequency domain as (3),

$$
S(f)=E\left(X(f) \cdot X^{*}(f)\right)
$$

In (3), $X(f)$ is the Fourier transform of $x(t)$. The * operator denotes complex conjugation and the $E()$ operator denotes the mean value of results from an ensemble of stationary periods of length $T_{\text {seg }}$. It is easy to read from (3) that the PSD indicates the spectrum distribution of power contained in the stochastic process, since the amplitude of Fourier transform is calculated by (3).

Nevertheless, the power of PSD is not only limited in spectrum analysis. There are 2 other properties of PSD bridging the WSS in time domain and frequency domain.

1) For WSS, if $\int_{-\infty}^{\infty}\left|R_{X}(\tau)\right| d \tau<\infty$, then PSD $S(f)$ is the Fourier transform of $R_{x}(\tau)$, which can be expressed by (4)

$$
S(f)=\int_{-\infty}^{\infty} R_{X}(\tau) e^{-j 2 \pi f \tau} d \tau
$$

2) For one linear system, the frequency response of which is $H(f)$, the PSD $S_{Y}(f)$ of output series $y(t)$ of this system is $S_{Y}(f)=|H(f)|^{2} S_{X}(f)$, by assuming the PSD of input series as $S_{X}(f)$.

Property 1) indicates the fluctuation of WSS can be equally described in frequency domain by PSD, since the power spectrum distribution $S(f)$ is the Fourier Transform of autocorrelation function $R_{x}(\tau)$. The time series of WSS varies every second, therefore it is not possible to observe a general stochastic rule in time domain. However, the PSD curve keeps almost constant for stationary process, so it is easy to identify the stochastic features of WSS in frequency domain. This transformation turns the original stochastic problem into the deterministic problem, significantly reducing the study difficulty. For property 2), the frequency response $H(f)$ is convenient to be calculated by transfer function, which may be the most common way to model any linear system. Based on that, 2) shows the stochastic output can be simply determined by the input PSD and $H(f)$ in frequency domain. This conclusion makes the existed mature models compatible with the wind power fluctuation research, rather than developing new models or methods to deal with the stochastic dynamic problem in time domain. These two properties of PSD build up the connection in time and frequency domain, mathematically explaining why the wind power fluctuation can be conveniently modeled in frequency domain.

\section{BASIC StRUCTURE OF WIND POWER FLUCTUATION MODEL}

The simulation model published in [12] uses a statistical description of the wind speed given in the frequency domain to simulate time series of output from a wind farm. In this section, a brief description is provided only to indicate the most critical procedures of simulation, and readers can find more details of the model in [12][14].

Fig. 1 shows the basic structure of the simulation model of power fluctuation from a wind farm with a number $\mathrm{N}$ of wind turbines. The PSD $S_{u[i]}(f)$ of the wind speed measured in the hub height of each wind turbine $i$ is given as input. A more general PSD model is proposed in [12], in order to fit the measurement result into a PSD function containing lowfrequency and high-frequency fraction. For each wind turbine, a rotor wind block calculates the PSD $S_{u[i]}(f)$ of an equivalent wind speed $u_{e q[i]}(t)$, which includes the smoothing of the wind speed due to the weighted averaging over the rotor.

The simulation results are also quite sensitive to the specification of the coherence functions. The coherence functions quantify the correlation between the wind speeds at the individual wind turbines. The definition of the coherence function $\gamma_{u[r, c]}(f)$ between $u_{[r]}(t)$ and $u_{[c]}(t)$ is given by

$$
\gamma_{u[r, c]}(f)=\frac{S_{u[r, c]}(f)}{\sqrt{S_{u[r]}(f) \cdot S_{u[c]}(f)}}
$$

$S_{u[r, c]}(f)$ is the cross power spectral density (CPSD) between between $u_{[r]}(t)$ and $u_{[c]}(t)$, defined according to

$$
S_{u[r, c]}(f) \Delta f=E\left(U_{[r]}(f) \cdot U_{[c]}^{*}(f)\right)
$$

The kernel of the model is the time series simulator. The first part of this simulator CPSD matrixes $\mathbf{S}(f)$, which takes the correlation between the wind speeds expressed by the 
coherence functions $\gamma_{[r, c]}(f)$ into account. The second part transforms the deterministic CPSD matrixes into stochastic time series $u_{e q[i]}(t)$ of the equivalent wind speeds. In [12], this transformation is implemented by a well-known Cholesky

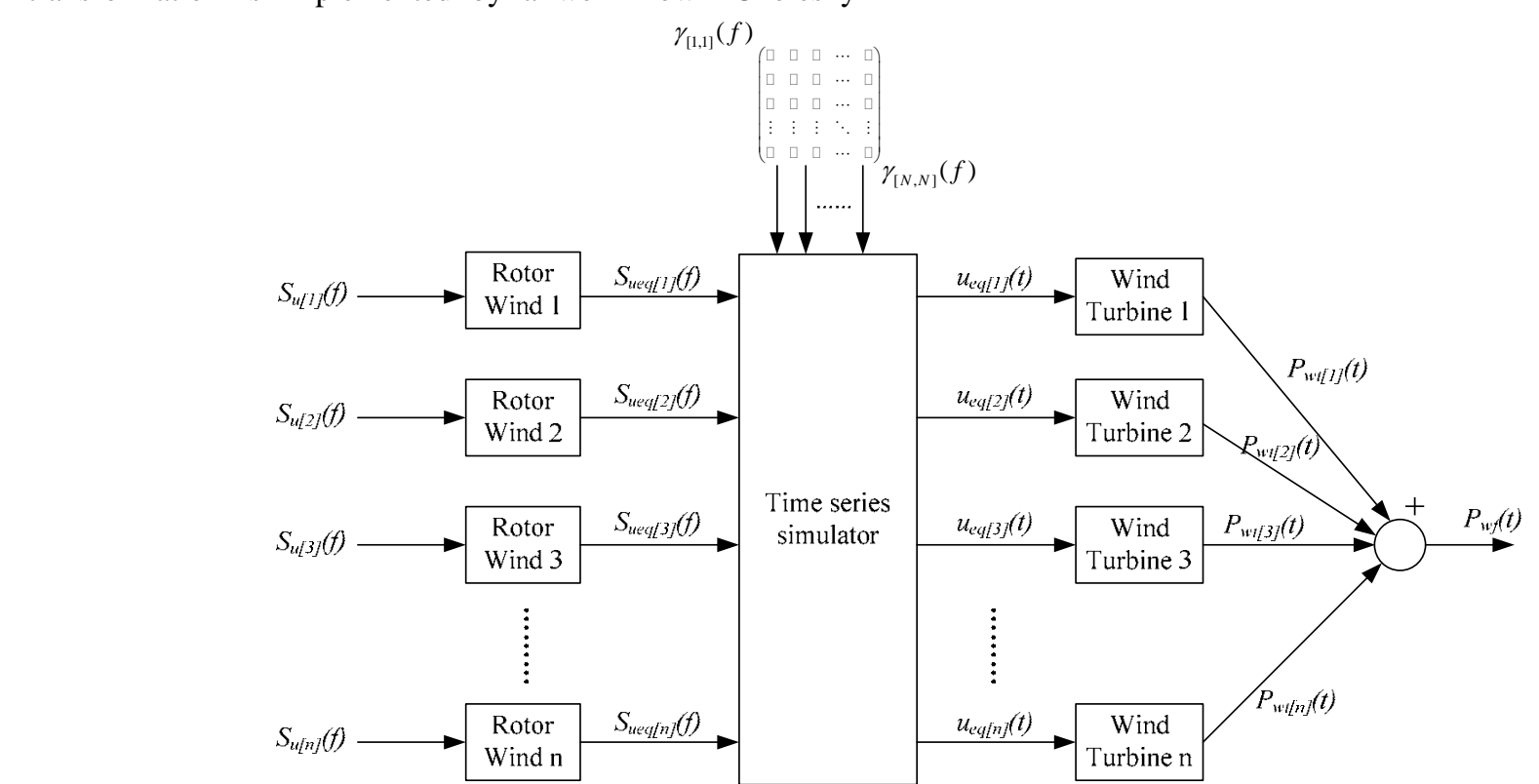

decomposition algorithm. The wind turbine blocks then calculate the produced electrical power $P_{w t[i]}(t)$. Finally, the power of all wind turbines is summed up to obtain the output power $P_{w f}(t)$ of the wind farm.

Fig. 1 Structure of model for simulation of power fluctuation from wind farm

\section{ENGineERING IMPLEMENTATION OF Simulation Model}

In Section III, the original model includes some frequency-time transitions, parts of which are timeconsuming, particularly when the computation loads of transitions increase rapidly if greater simulation precision is required or the scale of wind farm expands[16]. Actually the model in Fig. 1 is a completed theoretical model, but the engineering implementation of this model can be simplified to satisfy some special requirements of power system applications.

This section focuses on the simplification of CPSD matrixes of the power fluctuation model to accelerate the simulation of power fluctuation. The idea of this section is to reserve the CPSD matrixes in middle frequency region, but to simplify the CPSD matrixes in low and high frequency regions.

A. Feasibility of Cholesky decomposition in low frequency region

Mathematically, the CPSD matrixes are probably infeasible to be decomposed by Cholesky decomposition in low frequency region. It is because that the Cholesky decomposition strictly requires the matrix to be positivedefinition. Otherwise, if it contains any negative eigenvalues, there is no real root for the second equation of (7) given in [12]. That leads the decomposition failure.

$$
U_{L T[r, c]}= \begin{cases}\frac{S_{[r, c]}(f) \cdot 2 T-\sum_{i=1}^{c-1} U_{L T[r, i]} U_{L T[c, i]}}{U_{L T[c, c]}}, & c<r \\ \sqrt{S_{[r, r]}(f) \cdot 2 T-\sum_{i=1}^{r-1}\left|U_{L T[r, i]}\right|^{2}}, & c=r\end{cases}
$$

Physically, these indecomposable CPSD matrixes can be explained by the "tight coupling" between wind speeds in the low frequency region. The coherence function is modeled as (8) in [12],

$$
\gamma_{[r, c]}(f)=\exp \left(-\left(A_{r r, c]} \frac{d_{[r, c]}}{V_{0}}+j 2 \pi \tau_{[r, c]}\right) f\right)
$$

In (8), the first term inside the $\exp ()$ models the amplitude coherence, and the second term denotes the phase coherence. If the frequency of CPSD matrix is very low, the amplitude coherence is close to 1 , and the phase coherence is close to 0 . This implies the wind speeds of all wind turbines in this frequency region are tightly coupled together and fluctuate synchronously just like one single turbine. This kind of coupling can be denoted as a 1-full coherence matrix as (3),

$$
\gamma_{[r, c]}(f)=\left(\begin{array}{cccc}
1 & 1 & \cdots & 1 \\
1 & 1 & \cdots & 1 \\
\vdots & \vdots & \ddots & \vdots \\
1 & 1 & \cdots & 1
\end{array}\right)
$$

After the program detects that the current CPSD matrix is decomposable, it substitutes (9) into (8) to regenerate the CPSD matrix. The generated CPSD matrix is very special, which allows the lower-triangular matrix can be written directly as (10) and avoids the infeasible decomposition of 
(7). The physical explanation of (10) is all the wind speeds are modeled as fluctuating synchronously with the wind in the hub height of the $1^{\text {st }}$ wind turbine.

$$
U_{L T[r, c]}=\left(\begin{array}{cccc}
1 & 0 & \cdots & 0 \\
1 & 0 & \cdots & 0 \\
\vdots & \vdots & \ddots & \vdots \\
1 & 0 & \cdots & 0
\end{array}\right) \mathbf{S}_{\text {иеq }}(f)
$$

\section{B. Reduction of the CPSD matrixes in high frequency region}

In contrast with the case in low frequency region, the fluctuation of wind speeds of different turbines are "loosely" coupled together in high frequency region. This is supported by the statistical result from the test site in Høvsøre, Denmark. The results are published in [17] in 2005 and referred in this paper here as Fig. 2 and Fig. 3.
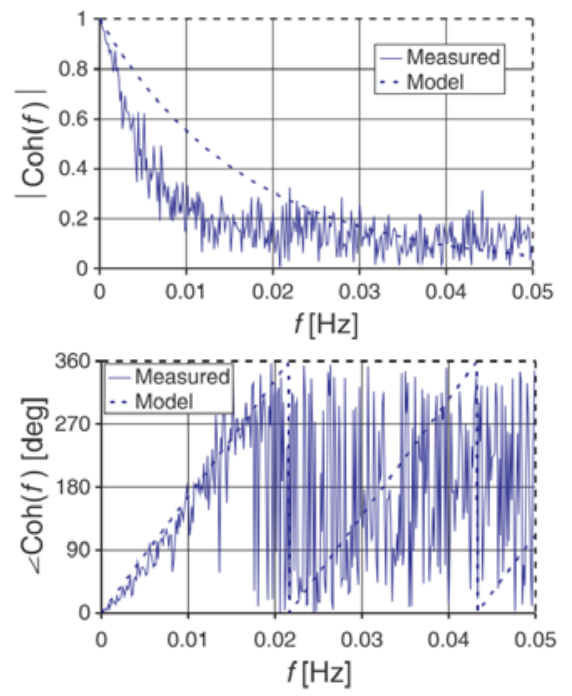

Fig. 2 Measured longitudinal coherence between wind speeds on 2 masts based on $2 \mathrm{~h}$ segments with mean wind speeds $4 \mathrm{~ms}^{-1}<\mathrm{V} 0<8 \mathrm{~ms}^{-1}$ (P. Søren[17])
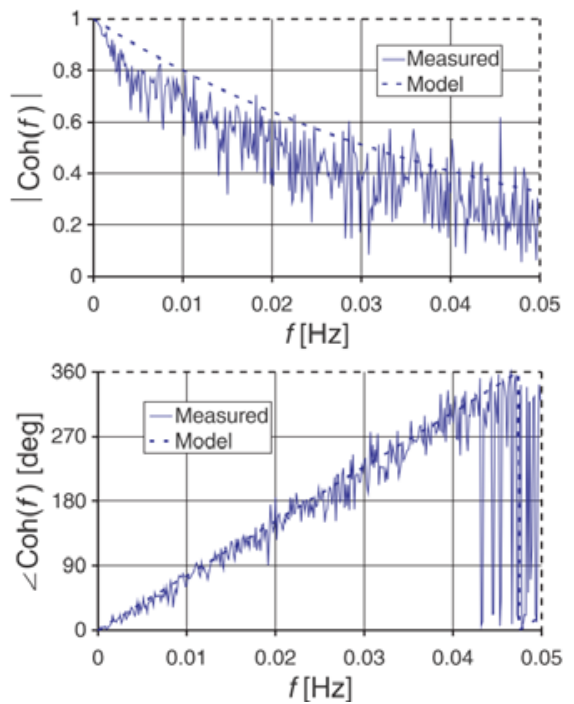

Fig. 3 Measured longitudinal coherence between wind speeds on 2 masts based on $2 \mathrm{~h}$ segments with mean wind speeds $12 \mathrm{~ms}^{-1}<\mathrm{V} 0<20 \mathrm{~ms}^{-1}$ (P. Søren[17])
From Fig.2 and Fig.3, it is easy to observe three characteristics of coherence of wind speeds in high frequency as following:

1) A "sign" frequency can be easily distinguished in the position where the coherence phase is 360 degrees. In Fig. 2, this "sign" frequency is around $0.021 \mathrm{~Hz}$, and in Fig. 3, it is around $0.048 \mathrm{~Hz}$;

2) Higher than the "sign" frequency, the amplitude coherence is near to 0 and the phase coherence becomes totally random;

3) Comparing Fig.2 and Fig.3, the "sign" frequency increases with the average wind speed.

The first two characteristics demonstrate the "loose coupling" between wind speeds in the high frequency region, especially when over the "sign" frequency. Therefore, it is reasonable to use a unit matrix as (11) to model this kind of "loose" coherence.

$$
\gamma_{[r, c]}(f)=\left(\begin{array}{cccc}
1 & 0 & \cdots & 0 \\
0 & 1 & \cdots & 0 \\
\vdots & \vdots & \ddots & \vdots \\
0 & 0 & \cdots & 1
\end{array}\right)
$$

The $3^{\text {rd }}$ characteristic implies that the "sign" frequency does not usually keep constant, and it needs to be updated according to different mean wind speed in each simulation segment. As similar as the case in low frequency region, after the program detects the "sign" frequency, the substitution of coherence functions of (11) helps the program avoid both of the generation and decomposition of CPSD matrixes, leading to a much faster simulation speed.

\section{VALidation of Simulation Model}

In order to verify the simulation model, the program is executed to simulate a one-month power fluctuation of a wind farm with 49 wind turbines. The simulation result is firstly verified by the coherence of equivalent wind speeds of wind turbines. Since in Section IV some simplifications have been designed to reduce the computation overhead, it is necessary to know how the simplified model looks like. The second validation is to compare the PSD of simulation result and field measurement, the data of which are from NingXia Grid Company. This validation is designed to test whether the simulation result still matches the reality after some model simplification introduced.

A. Validation of the coherence of equivalent wind speeds of wind turbines

The simulation results from the $1^{\text {st }}$ and $2^{\text {nd }}$ turbine are selected to obtain the equivalent wind speeds coherence, which are depicted in Fig.4. In the low frequency region of Fig.4, the amplitudes of $2^{\text {nd }}$ and $3^{\text {rd }}$ coherence are equal to 1.0. Comparing the original model plotted by red line, the result of Fig. 4 indicates the $2^{\text {nd }}$ and $3^{\text {rd }}$ CPSD matrixes are simplified. The fluctuations are assumed to be aggregated in a single point of turbine 1 . In fact, although this assumption really increases the simulated power fluctuation in the low frequency region, this simplification is still inevitable due to 
the infeasibility of Cholesky decomposition of these CPSD matrixes. And the model amplitude coherence is near to 1 , so the error led by this simplification is not far away from reality.
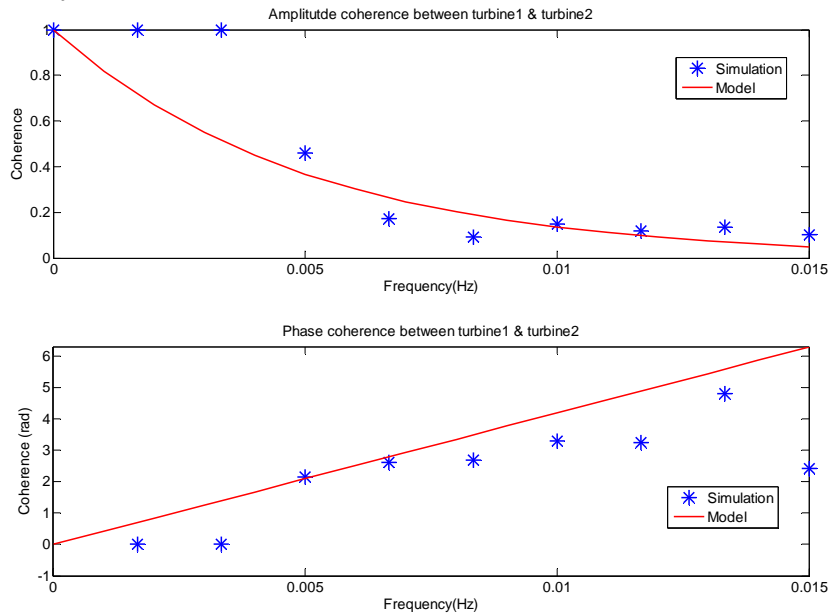

Fig. 4 Coherence of power fluctuation between turbine 1 and turbine 2 before and after low frequency simplification

\section{B. Validation of the PSD of power fluctuation of wind farm}

The simulation result is compared with the field measurement by PSD in Fig.5. In this figure, the simulation result (blue line) matches the measurement (red line) very well, which shows the errors induced by the model simplification do not significantly affect the validity of simulation.

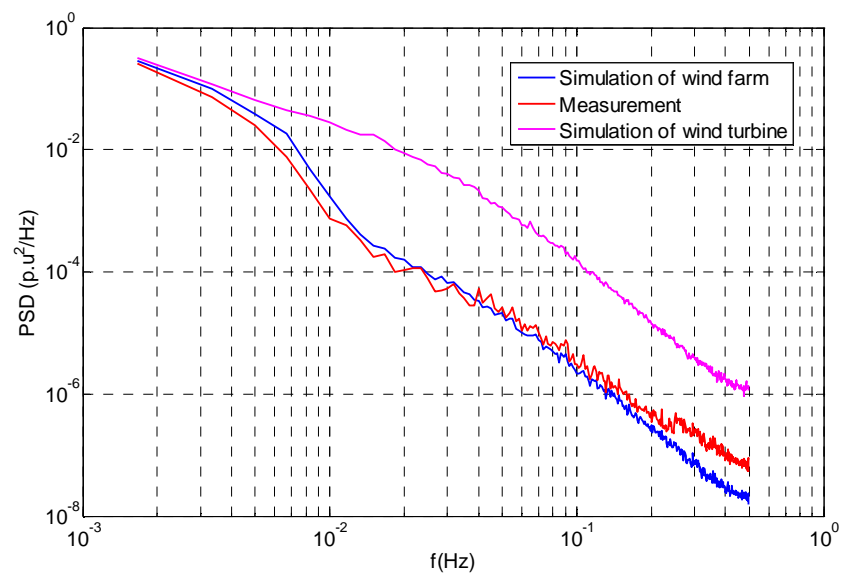

Fig. 5 Validation of the PSD of power fluctuation of wind farm

This result is also verified by comparing the PSD of wind farm and wind turbine. In low frequency region, the pink line is close to the blue line, because the wind speeds of wind turbines are tightly coupled. But in high frequency region, the power fluctuation from one single wind turbine is much higher than that from one wind farm since the wind speed of each wind turbine is considered as independent, leading to the "Effect of geographical dispersion"[1]. Both of them prove the engineering implement in Section IV works quite well to match the real world. Finally, one important thing to note here is that the PSD curve of power fluctuation which is simulated without the model simplification is not plotted in Fig.5. The only reason for that is this curve is so close to the PSD curve simulated with model simplification, that it is almost impossible to be distinguished if both of them are plotted together.

\section{Applications of Simulation Model on Power SYSTEMS}

The impact of imbalance due to wind power fluctuation is the increased power system ramp rate and reserve requirements. It is critical to evaluate these changes in order to plan and operate the power systems safely and economically. In [13], the definition of ramp rates and reserve requirements are involving a statistical period time $T_{p e r}$, which reflects the time scale of interest. The ramp rate is simply the change in mean value from one period $T_{p e r}$, as (12),

$$
P_{\text {ramp }}(n)=P_{\text {mean }}(n+1)-P_{\text {mean }}(n)
$$

Note that this definition specifies the ramping of the wind farm power. Thus, negative ramp rate means decreasing wind power, which requires positive ramping of other power plants.

The intention of reserve requirements is to quantify the difference between the instantaneous power and the mean value that are dealt with as ramping. Since the reserves must be allocated in advance, the positive reserve requirement is defined as the difference between the initial mean value and the minimum value in the next period. Formally, the reserve requirements are defined as (13)

$$
P_{\text {res }}(n)=P_{\text {mean }}(n)-P_{\min }(n+1)
$$

Note that with this definition, positive reserves mean decreasing wind power that requires positive reserves from other power plants.

The illustrations of both of these definitions can be found in [13].

When the ramp rates and reserve requirement have been calculated for each set of neighbor periods, both of them are binned according to the corresponding initial power. This is because the statistics of the ramping and reserve will depend strongly on the initial power. For instance, the power is not likely to increase very much when it is already close to rated under normal weather condition. After the ramping is sorted in each power bin, a duration curve is obtained.

The most interesting point of system operation is the highest requirement to the ramp rates and reserve requirement of other power plants is quantified. They are the $1 \%$ and $99 \%$ percentile respectively in the ramp rates and reserve duration curves. System operators can find the quantification in each power bin to make the optimal decision for next operation period time.

\section{A. Ramp rates and reserve requirement under normal weather condition}

The wind speed is lower than cut-out speed under normal weather condition. The wind farm operates normally without emergency protection, if no physical accident occurs. This kind of scenario usually occupies over $90 \%$ of wind farm site 
conditions, so the case study here can help the system operators handle the routine operations, such as day-ahead generation schedule.

The simulation results of wind farm and wind turbine, as well as the measurement results are compared from Fig. 6 to Fig.9. The duration curves are plotted in Fig.6 and Fig.7, where the initial power range from $0.7-0.8$ p.u.. The $99 \%$ and $1 \%$ percentile are also pointed out by arrows in these figures. Then the Fig.8 and Fig.9 connect all the percentiles points together showing the maximum ramp rates and reserve requirements. These figures can give the maximum estimation to the system operators for the integration of large wind farm.

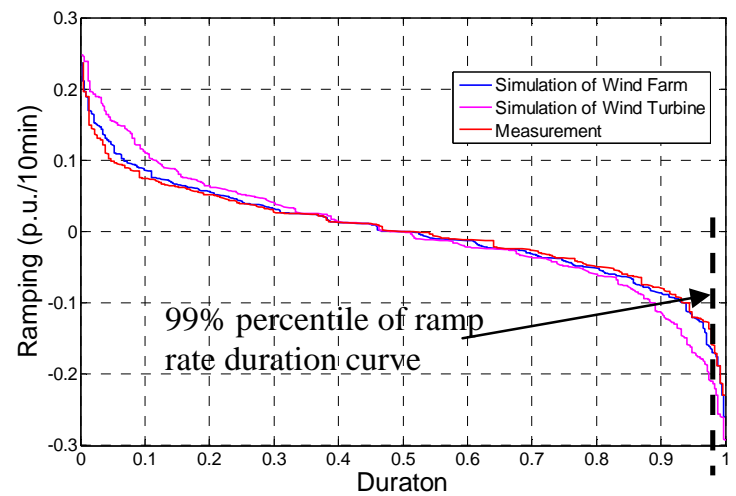

Fig. 6 Duration curves of 10-min ramp rates in the initial power range from 0.7 p.u. to 0.8 p.u.

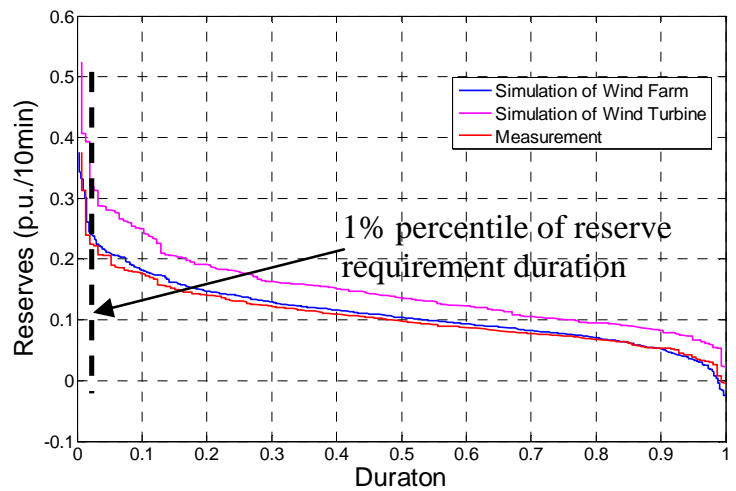

Fig. 7 Duration curves of 10 -min reserve ramps in the initial power range from 0.7 p.u. to 0.8 p.u.

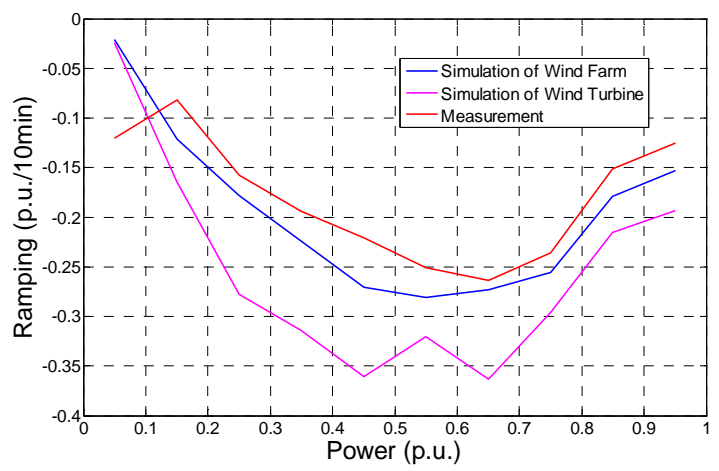

Fig. 8 The 99\% percentiles of 10 -min ramp rates in all power ranges

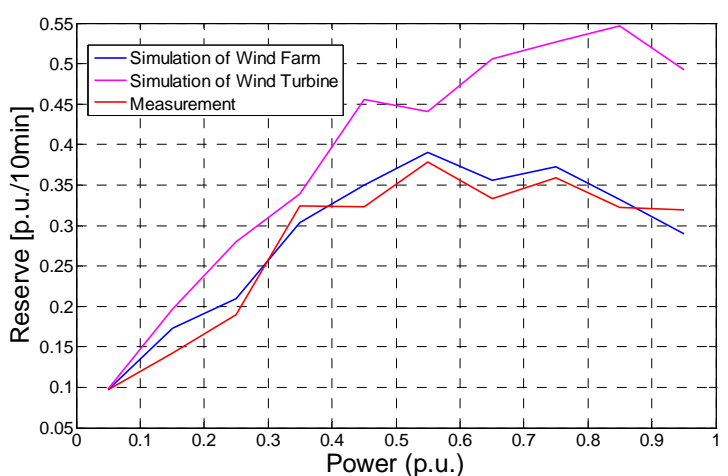

Fig. 9 The $1 \%$ percentiles of 10 -min reserve requirements

In Fig.6 Fig.9, the simulation results agree very well to the measurement statistical curves. This verifies the model. As a comparison, the simulation result of single wind turbine shows more fluctuation than measurement. It is because the single-aggregated model cannot take the effect of dispensation into account, and result in a worse estimation.

Another interesting point is that both of the curves in Fig.8 and Fig.9 show a tendency to increase until 0.9 p.u. and then decrease a little in the last power bin. This is because the wind farm ramps and fluctuates less for wind speeds above rated, where the wind turbines are working on the flat part of the power curve under the normal weather condition.

B. Ramp rates and reserve requirement under extreme weather condition

Wind turbines suffer extremely high wind speed under this weather condition. Once the wind speed is higher than cutout speed, wind turbine has to emergently brake to avoid physical damages. The sudden loss of power can intensify the fluctuation of wind farm, making troubles for the system operators. The model proposed in this paper is capable to simulate the wind fluctuation under extreme weather condition. The simulated time series are depicted in Fig.10 and Fig.11.

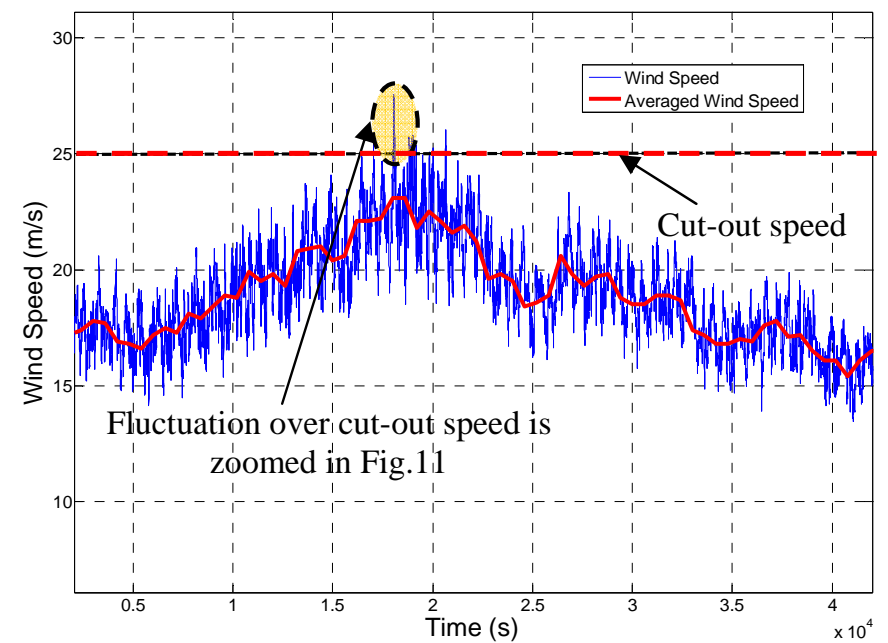

Fig. 10 The simulated wind speed series under extreme weather condition 


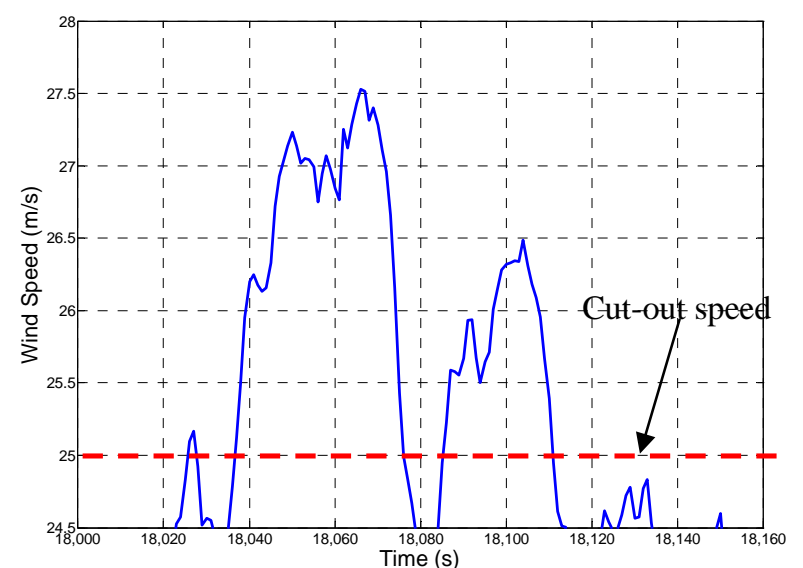

Fig. 11 The zoomed wind fluctuation over cut-out speed

In Fig.10, the red curve is the 10-min averaged wind speed, which is obviously below the cut-out speed $(25 \mathrm{~m} / \mathrm{s})$. However, the real-time wind (the blue curve) is beyond the cut-out speed once the fluctuation is taken into account. Fig.11 is a zoomed view of the wind fluctuation between 18000s and 18160s. There are longer than 60s that the realtime wind is clearly over the cut-out speed. By this higher resolution, the model helps to give more information for the power fluctuation simulation of wind farm shown in Fig.12 and Fig.13.
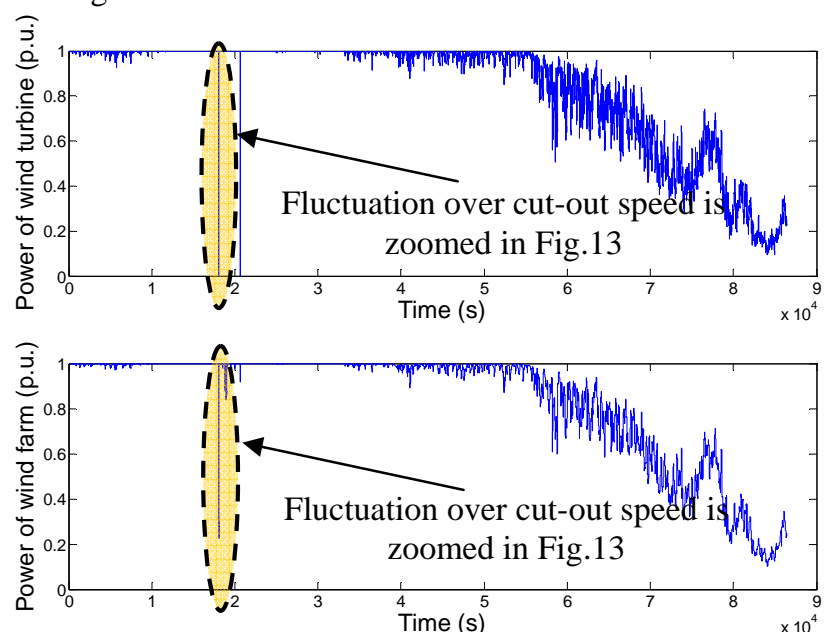

Fig. 12 Power fluctuation series simulated by wind turbine and wind farm
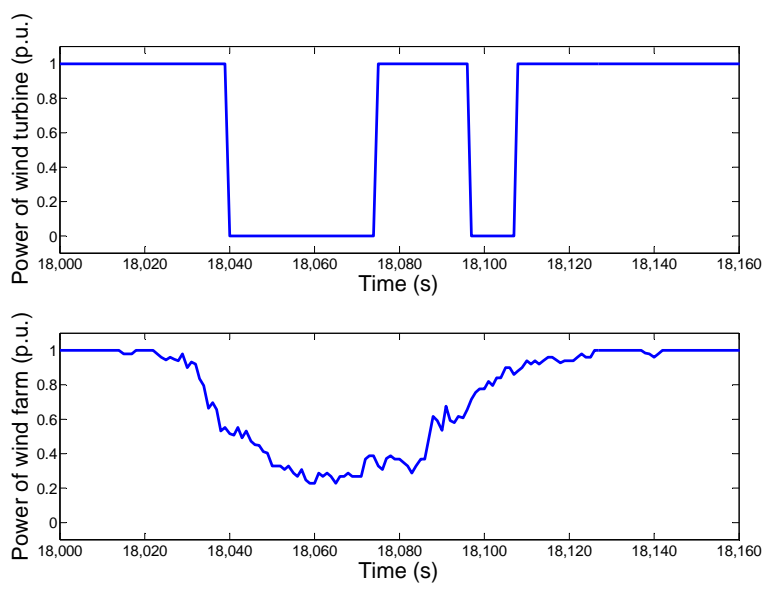

Fig. 13 The zoomed power fluctuation over cut-out wind speed
The power fluctuations from wind turbine and wind farm are plotted respectively in Fig.12. The first glance is the fluctuation of wind turbine (upper subfigure) is much higher than wind farm (lower subfigure) since the "Effect of geographical dispersion". A further finding is the sudden power loss simulated in the yellow area. This is due to the real-time wind speed has gone beyond the cut-out speed, and the wind turbines have to be out of service to protect themselves. Note that this sudden power loss is impossible to be simulated by a 10-min resolution. It occurs only after the simulation resolution is increased, and the fluctuation within 10 mins is subsequently considered.

Fig.13 plots a zoomed view of one part of the sudden power loss. The behaviors of wind turbine and wind farm look different during power loss. The power of single wind turbine drops steeply but the wind farm shows a much slower decline. This can be explained by the layout of wind farm. Though one wind turbine stops once the front of high wind hits it, all of them do not stop simultaneously since the front still needs time to pass through the distance between wind turbines. This simulation result once again proofs the "Effect of geographical dispersion” has been perfectly included in this paper's model.

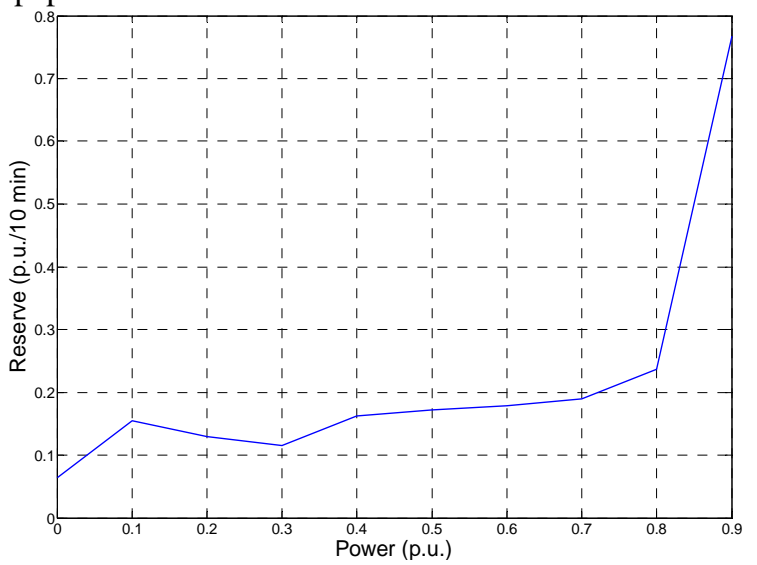

Fig. 14 The $1 \%$ percentiles of 10 -min reserve requirements under extreme weather condition

The $1 \%$ percentiles of 10 -min reserve requirements are shown in Fig.14. Compared with Fig.9, it is clear that the tendency is a sharply increase in the last power bin. That means system operators should seek for more reserve resources to meet the possibility that wind turbines are cut out under extreme weather condition.

Frankly speaking, there is still some untrue while the model runs under high wind speed. It is impossible for wind turbines to take such a rapid power recovery in Fig.13. It usually requires minutes at least. This defect is because the start-up time of wind turbine has not been modeled in the "Wind Turbine" Block of Fig.1. Therefore the lowest point of Fig.13 should be around 0 p.u., and the reserve requirements in Fig.14 should be around 1 p.u. as all wind turbines should stay offline at that time.

However, this defect does not influence any analysis above, especially the slower power loss due to geographical dispersion. This is still a very detailed and precise model to depict the power fluctuation of large wind farm. And some future work can definitely fix this start-up time problem. 


\section{CONCLUSION}

This paper proposes a model in frequency domain. The first reason to choose frequency domain is the fluctuation in time domain can be equally described in frequency domain by PSD. PSD connects the fluctuation stochastic with linear system, which makes the existed mature models compatible with the wind power fluctuation research.

An engineering implement is also presented in this paper. The idea is to reserve the CPSD matrixes in middle frequency region, but to simplify the CPSD matrixes in low and high frequency regions to accelerate the simulation of power fluctuation. The simplified model is verified by coherence and PSD analysis, which show the error led by this simplification is not far away from reality.

Finally, this model is applied on power system studies on how to arrange the system ramp rates and reserve efficiently. Under normal weather condition, the results show a tendency to increase until 0.9 p.u. and then decrease a little in the last power bin. This is because the wind farm ramps and fluctuates less for wind speeds above rated. Under extreme weather condition, a higher resolution provided by this model helps to simulate a sudden power loss due to the emergency protection of wind turbines. Furthermore the "Effect of geographical dispersion" is observed from the slower decline of wind farm during power loss. This simulated time series give the hints to system operators to seek for more reserve resources to meet the possibility that wind turbines are cut out under extreme weather condition.

In conclusion, even small defect existing, this model is still a very detailed and precise model to depict the wind power fluctuation. It is also very powerful and flexible to be applied on power system studies, in order to support the economical balance operations of power systems

\section{ACKNOWLEDGEMENT}

This research work was supported by the Chinese National Natural Science Funds (50977050, 50823001). The authors gratefully acknowledge the support of BP Alternative Energy Inc on this project.

\section{REFERENCES}

[1] F. Van Hulle, "Large Scale Integration of Wind Energy in the European Power Supply, Analysis, Issues and Recommendations", EWEA Tech Rep, pp. 52-59, 2005, [Online]. Available: http://www.ewea.org/fileadmin/ewea_documents/documents/publicatio ns/grid/051215_Grid_report.pdf

[2] Hadi Banakar, Changling Luo and Boon Teck Ooi, "Impacts of Wind Power Minute-to-Minute Variations on Power System Operation”, IEEE Trans. Power Systems, vol. 23, pp. 150-160, Feb. 2008.

[3] Pei Li, Hadi Banakar, Ping-Kwan Keung, Hamed Golestani Far, and Boon-Teck Ooi, "Macromodel of Spatial Smoothing in Wind Farms", IEEE Trans. Energy Conversion, vol. 22, pp. 119-128, Mar. 2007.

[4] Yi Gao, R. Billinton, "Adequacy assessment of generating systems containing wind power considering wind speed correlation”, IET Renewable Power Generation, vol. 3, pp. 217-226, 2009.

[5] Roy Billinton and Yi Gao, "Multistate Wind Energy Conversion System Models for Adequacy Assessment of Generating Systems Incorporating Wind Energy”, IEEE Trans. Energy Conversion, vol. 23, pp. 163-170, Mar. 2008.

[6] Ronan Doherty and Mark O’Malley, “A New Approach to Quantify Reserve Demand in Systems With Significant Installed Wind
Capacity”, IEEE Trans. Power Systems, vol. 30, pp. 587-595, May 2005.

[7] Aidan Tuohy, Peter Meibom, Eleanor Denny and Mark O'Malley, "Unit Commitment for Systems With Significant Wind Penetration", IEEE Trans. Power Systems, vol.24, pp.592-601, May 2009.

[8] Changling Luo and Boon-Teck Ooi, "Frequency Deviation of Thermal Power Plants Due to Wind Farms", IEEE Trans. Power Delivery, vol.21, pp. 708-716, Sep. 2006.

[9] Changling Luo, Hamed Golestani Far, Hadi Banakar, Ping-Kwan Keung, and Boon-Teck Ooi, "Estimation of Wind Penetration as Limited by Frequency Deviation", IEEE Trans. Energy Conversion, vol.22, pp. 783-791, Sep. 2007.

[10] Söder L, Holttinen H., "On methodology for modelling power system impact on power systems”, International Journal of Global Energy, vol.29, pp. 181-198, 2008.

[11] P. Sørensen, A.D. Hansen, P.A.C. Rosas, "Wind models for simulation of power fluctuations from wind farms", Wind Engineering Industry Aerodynamic, no.90, pp. 1381-1402, 2002.

[12] P. Sørensen, Nicolaos Antonio Cutululis and A.D. Hansen, "Modelling of Power Fluctuations from Large Offshore Wind Farms", Wind Energy, vol. 11, pp. 29-43, 2008.

[13] Poul Sørensen, Nicolaos Antonio Cutululis and Antonio ViguerasRodríguez, "Power Fluctuations From Large Wind Farms", IEEE Trans. Power Systems, vol. 22, pp. 958-965, Aug. 2007.

[14] Poul Sørensen, Pierre Pinson, Nicolaos Antonio Cutululis, "Power fluctuations from large wind farms", Risø-R-1188(EN). Roskilde, Aug. 2009.

[15] LIN Jin, SUN Yuan-zhang and Poul Sørensen, "Modeling and Simulation of power fluctuation of large wind farm based on frequency domain”, Automation of Electric Power Systems, Submitted.

[16] LIN Jin, SUN Yuan-zhang, Poul Sørensen, LI Guo-jie, LI Xiong, "Fast simulation approaches for power fluctuation model of large wind farm", Wind Engineering and Industrial Aerodynamics, Submitted.

[17] P. Sørensen, J. Mann, U.S. Paulsen and A. Vesth, "Wind Farm Power Fluctuations", Chapter 25 of Wind Energy Proceedings of the Euromech Colloquium, Springer Berlin Heidelberg, pp. 139-145, 2007.

\section{BiographiES}

LIN Jin received his Bachelor degree in Electrical Engineering from Tsinghua University, China in 2007. He then continues to pursue a Ph.D. degree in the same department. Currently, he is sponsored by Chinese Scholarship Council to be a visiting student in Risø Laboratory, Roskilde, Denmark. He is co-supervised by Poul Sørensen in Risø working on the power fluctuation of wind farm. His research interests are the integration study of large wind power and power system simulation technology.

SUN Yuan-zhang (M'99-SM'01) received the B.S. degree from Wuhan University of Hydro and Electrical Engineering, China, the M.S. degree from Electric Power Research Institute (EPRI), China, and Ph.D. degree from Tsinghua University, Beijing, in 1978, 1982, and 1988, respectively, all in electrical engineering. He is now a chair professor at Tsinghua University and dean of School of Electrical Engineering of Wuhan University. His main research interests are in the areas of power systems dynamics and control, voltage stability and control, and renewable energy.

Poul Sørensen (M’04-SM’07) was born in 1958. He received the M.Sc degree in electrical engineering from the Technical University of Denmark, Lyngby, in 1987. Since 1987, he has been employed at Risø National Laboratory, Roskilde, Denmark, presently as a Senior Scientist. Now he is a professor in Risø. His main technical interest is integration of wind power into power systems, involving a variety of technical disciplines including power system control and stability, dynamic modeling and control of wind turbines and wind farms, and wind fluctuation statistics.

LI Guo-jie received his Bachelor and Master degrees in Electrical Engineering from Tsinghua University, P.R. China in 1989 and 1993, respectively. He also received $\mathrm{Ph}$. D. degree in the School of Electrical and Electronic Engineering, Nanyang Technological University Singapore in 1999. He is now an associate professor in the Department of Electrical Engineering, Tsinghua University, P. R. China. His current research interests include power system analysis and control, wind power analysis and control, and power quality. 